\title{
Autologous Stem Cell Transplantation in Testicular Germ Cell Tumor-Preliminary Experience from a Single Center
}

\author{
Gangothri Selvarajan ${ }^{1}$ Perumal Kalaiyarasi Jayachandran ${ }^{1}$, Arun Kumar Rajan ${ }^{1}$ Sivashree Kesana ${ }^{1}$ \\ Krishnarathinam Kannan ${ }^{1}$ Tenali Gnana Sagar ${ }^{1}$ Trivadi Sundaram Ganesan \\ ${ }^{1}$ Department of Medical Oncology, Cancer Institute (WIA), Chennai, \\ Tamil Nadu, India \\ Address for correspondence Perumal Kalaiyarasi Jayachandran, MD, \\ MRCP (UK), DM, Department of Medical Oncology, Cancer Institute \\ (WIA), 38, Sardar Patel Road, Guindy, Chennai, Tamil Nadu,India \\ -600036, (e-mail: dr.pkjayachandran@gmail.com).
}

South Asian J Cancer 2021;10:97-101.

\section{Abstract

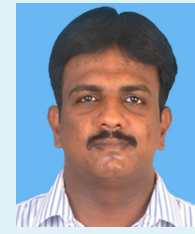 \\ Perumal Kalaiyarasi Jayachandran \\ Keywords \\ - relapsed germ cell tumor \\ - autologous stem cell transplant \\ - high-dose chemotherapy \\ - high-dose carboplatin and etoposide \\ - long-term survival in relapsed GCT}

Background Germ cell tumor (GCT) of the testis is one of the highly curable solid organ malignancies. Those who experience relapse after platinum-based chemotherapy can be salvaged with systemic therapy followed by high-dose chemotherapy (HDCT) and autologous stem cell transplantation (ASCT). Complete remission can be obtained in approximately 50 to $60 \%$ of patients treated with HDCT. Our experience reports the efficacy and safety of HDCT followed by ASCT in relapsed GCT.

Methods Analysis of patient records (2012-2019) showed that three patients had received HDCT and ASCT.

Results All the three patients were treated with BEP (bleomycin, etoposide, and cisplatin) as first-line therapy. HDCT was done in Case 1 after third-line salvage and in other two patients after second-line salvage chemotherapies. High-dose carboplatin and etoposide were used as conditioning regimen. Granulocyte colony-stimulating factor was used for the mobilization of stem cells. After ASCT, complete remission was documented in all the patients. All were alive and disease-free till the last follow-up. Grade $3 / 4$ toxicities including myelosuppression, diarrhea, and mucositis were observed in all three patients.

Conclusion This is the first report from India on HDCT with ASCT in GCT. HDCT/ASCT seems to be feasible, safe, and effective in relapsed testicular GCTs.

\section{Introduction}

Germ cell tumor (GCT) of the testis is one of the highly curable solid organ malignancies despite the presence of disseminated disease. Testicular cancer commonly affects males between the ages of 15 and 40 years. They are highly sensitive to platinum-based chemotherapy. Since the use of cisplatin, the cure rates have reached 90 to $95 \%$. None of the other advanced solid tumor malignancies have such high survival. The 5-year disease-free survival of patients

\section{DOI https://doi.org/10.1055/s-0041-1731516 ISSN 2278-330X}

How to cite this article: Selvarajan G, Jayachandran P. K, Rajan A. K, Kesana S, Kannan K, Sagar T. G, Ganesan T. S. Autologous Stem Cell Transplantation in Testicular Germ Cell Tumor-Preliminary Experience from a Single Center. South Asian J Cancer 2021;10(2):97-101. with good, intermediate, and poor-risk disease are 89, 75, and $41 \%$, respectively. ${ }^{1,2}$ Cure rates are still lower in relapsed and refractory patients. Those who experience relapse after platinum-based chemotherapy can be salvaged with another course of platinum-based therapy followed by high-dose chemotherapy (HDCT) and autologous stem cell transplant (ASCT). ${ }^{3}$

The outcomes of the HDCT may vary from patient to patient. There are two prognostic scoring systems, namely, the Lorch-Beyer score (LBS) and Einhorn score, of which

(c) 2021. MedIntel Services Pvt Ltd.

This is an open access article published by Thieme under the terms of the Creative Commons Attribution-NonDerivative-NonCommercial-License, permitting copying and reproduction so long as the original work is given appropriate credit. Contents may not be used for commercial purposes, or adapted, remixed, transformed or built upon. (https://creativecommons.org/licenses/by-nc-nd/4.0/).

Thieme Medical and Scientific Publishers Private Ltd A-12, Second Floor, Sector -2, NOIDA -201301, India 
the former one is widely accepted. The benefit of HDCT reduces with increasing risk with both the scoring systems. In the International Prognostic Factors Study Group analysis, patients who had progressed after first-line cisplatin-based chemotherapy had a longer 2-year progression-free survival (PFS) (49.6\% vs. $27.8 \%$ ) and 3 -year overall survival (53.2\% vs. $40.8 \%$ in the HDCT group compared with the standard-dose chemotherapy group. ${ }^{4}$ The LBS was devised based on this report and four risk groups were identified. The benefit was seen across all risk categories but the magnitude of benefit in survival was high in the very low-risk category $(>70 \%)$ compared with the high-risk category (10-15\%)., In another report, retrospective analysis of patients who were refractory or received two or more lines of chemotherapy, and a scoring system was devised with three risk groups. This study also showed that in patients with intermediate-risk and high-risk score, the 2-year PFS were 60 and $40 \%$, respectively. ${ }^{6}$

With the availability of newer drugs like plerixafor which increases stem cell mobilization and advances in supportive care following HDCT, remission can be achieved in approximately 50 to $60 \%$ of relapsed patients treated with HDCT. ${ }^{7}$ There is no published literature from India in this setting. Hence, we present the preliminary experience from our center.

\section{Methods}

Data of adult patients with GCT of the testis, who had undergone stem cell transplant from January 2012 to December 2019, were captured from the patient records.

\section{Results}

A total of three patients underwent HDCT and ASCT for relapsed GCT during the study period. Baseline patient and treatment characteristics are shown in - Table 1. Granulocyte colony-stimulating factor was used for the mobilization of stem cells. The conditioning regimen used for HDCT was carboplatin at area under the curve 7 daily and etoposide $750 \mathrm{mg} / \mathrm{m}^{2}$ daily for 3 days (days $-4,-3,-2$ ). The toxicity following HDCT and ASCT are shown in - Table 2. The data were censored up to December 2019.

\section{Case 1}

A 24-year-old male patient presented with left testicular swelling and back pain. He underwent left high inguinal orchiectomy and was diagnosed as a non-seminomatous GCT of the testis (NSGCT) - stage IIIB with International Germ Cell Cancer Collaborative Group (IGCCCG) classification good risk. He received three cycles of bleomycin, etoposide, and cisplatin (BEP). His markers normalized after two cycles. Following chemotherapy, computerized tomography (CT) scan showed residual retroperitoneal and mediastinal lymphadenopathy. Hence, he underwent retroperitoneal lymph node dissection (RPLND) which had no viable tumor in the specimen. He was kept on follow-up. After 4 months, there was a rise in $\beta$-human chorionic gonadotropin ( $\beta$-HCG). CT scan had shown left para-aortic lymph nodal mass. He received four cycles of vinblastine, ifosfamide, and cisplatin (VeIP) chemotherapy regimen and had a complete response and hence was kept under follow-up. After 5 months, he had a recurrence again in the left para-aortic and left suprarenal region with rising alpha-fetoprotein (AFP) and $\beta$-HCG. He received two cycles of irinotecan, paclitaxel, and oxaliplatin (IPO) regimen $^{8,9}$ following which his tumor markers normalized and then he underwent excision of the left suprarenal nodal mass and left adrenalectomy. The histopathology was suggestive of a mixed GCT. He belonged to a very low-risk category as per the LBS. He received high-dose carboplatin and etoposide (HD-CE) followed by ASCT. The patient had febrile neutropenia and grade 4 mucositis during the procedure which was managed conservatively with crystalloids, total parental nutrition, and intravenous antibiotics. Neutrophil and

Table 1 Patient characteristics

\begin{tabular}{|l|l|l|l|}
\hline & Case 1 & Case 2 & Case 3 \\
\hline Age at diagnosis (in y) & 24 & 31 & 31 \\
\hline Age at transplant (in y) & 27 & 33 & 33 \\
\hline IGCCCG risk & Good & Poor & Intermediate \\
\hline Prior regimens chemotherapy before ASCT & 3 & 2 & 2 \\
\hline Einhorn risk score & Intermediate & High & Intermediate \\
\hline Lorch-Beyer risk score & Very low risk & High & Intermediate \\
\hline Conditioning regimen & HD-CE & HD-CE & HD-CE \\
\hline Mobilization regimen & G-CSF & G-CSF & 1 \\
\hline No of days of collection & 1 & 1 & 4.06 \\
\hline CD 34 cells (x10 cells/kg) & 3.6 & 5.5 & $11 / 17$ \\
\hline $\begin{array}{l}\text { Day of engraftment } \\
\text { neutrophil/platelets (in d) }\end{array}$ & $14 / 17$ & $19 / 22$ & 33 \\
\hline DFS (mo) till last follow-up & 39 & 22 & \\
\hline
\end{tabular}

Abbreviations: ASCT, autologous stem cell transplantation; DFS, disease-free survival; G-CSF, granulocyte colony-stimulating factor; HD-CE, high-dose carboplatin and etoposide; IGCCCG, International Germ Cell Cancer Collaborative Group. 
Table 2 Toxicity following high-dose chemotherapy (HDCT)

\begin{tabular}{|l|l|l|l|}
\hline Toxicities & Case 1 & Case 2 & Case 3 \\
\hline Neutropenia & Grade 4 & Grade 4 & Grade 4 \\
\hline Febrile neutropenia & Yes & Yes & Yes \\
\hline Thrombocytopenia & Grade 4 & Grade 4 & Grade 4 \\
\hline Anemia & Grade 3 & Grade 4 & Grade 1 \\
\hline Diarrhea & Nil & Grade 2 & Grade 3 \\
\hline Vomiting & Grade 1 & Grade 2 & Grade 2 \\
\hline Mucositis & Grade 3 & Grade 3 & Grade 3 \\
\hline Neurotoxicity & Nil & Nil & Nil \\
\hline Ototoxicity & Nil & Nil & Nil \\
\hline Nephrotoxicity & Nil & Nil & Nil \\
\hline Hepatotoxicity & Nil & Nil & Nil \\
\hline Pulmonary toxicity & nil & nil & Nil \\
\hline Secondary cancers & Nil & Nil & Nil \\
\hline
\end{tabular}

aPreexisting neuropathy was not aggravated.

platelet engraftments were observed on the 14th and 17th day, respectively. He is on active follow-up without disease for the past 39 months.

\section{Case 2}

A 31-year-old male patient presented with left testicular swelling and underwent a high inguinal orchiectomy. He was diagnosed as NSGCT stage IIIB with IGCCCG intermediate risk. He received four cycles of BEP and then he underwent RPLND followed by staged left lung metastasectomy. There was no viable tumor and he was kept on follow-up. After 1 month, he had rising markers and was found to have a recurrence in the liver and mediastinal lymph nodes. Following two cycles of second-line IPO chemotherapy, he developed grade 3 peripheral neuropathy and hence the chemotherapy was changed and two more cycles of etoposide, ifosfamide, and cisplatin (VIP) regimen were administered. Evaluation by CT scans showed the persistence of mediastinal lymph nodes with few calcifications. Markers were normal. He belonged to the high-risk category as per LBS. Subsequently, he underwent HDCT (HD-CE regimen) and ASCT. He developed grade 2 chemotherapy-induced nausea and vomiting (CINV), grade 2 diarrhea, and grade 3 mucositis which were managed conservatively with appropriate supportive care. Neutrophil and platelet engraftments were observed on the 19th and 22nd day, respectively. He is on active follow-up without disease for the past 22 months.

\section{Case 3}

A 31-year-old male patient presented with right testicular swelling and underwent high inguinal orchiectomy. He was diagnosed as NSGCT stage IIIB with IGCCCG intermediate risk. He received four cycles of BEP and had residual disease in the retroperitoneum with normal markers and hence underwent RPLND. There was no viable tumor. The AFP levels started rising serially after 6 months without detectable disease on imaging. He received one cycle of IPO as second-line regimen. He developed grade 3 diarrhea and hence further therapy changed to paclitaxel, ifosfamide, and cisplatin (TIP) regimen and he received three cycles. CT scan showed no evidence of any disease with normal markers. He belonged to the intermediate-risk category as per LBS. He received HDCT (HD-CE regimen) and ASCT. The patient developed grade 2 CINV, grade 3 diarrhea, electrolyte disturbances, febrile neutropenia, and grade 3 mucositis which were managed conservatively with appropriate supportive care. Neutrophil and platelet engraftments were observed on the 11th and 17 th day, respectively. He is on active follow-up without disease for the past 33 months.

\section{Discussion}

Although GCT is much less common in India, ${ }^{10}$ the proportion of patients with poor-risk disease at presentation is higher and their overall survival is only around $45 \%$. Therefore, there will be a cohort of patients who would benefit from more intensive treatment following a recurrence. ${ }^{11}$ However, to our knowledge, there are no published reports from India evaluating HDCT and ASCT in this setting.

Patients with recurrent GCT may still be cured in more than $50 \%$ of cases with salvage chemotherapy while it is only 10 to $20 \%$ among the high-risk group according to the LBS score. Usage of HDCT and ASCT as consolidation after initial first-line therapy in randomized control trials (RCTs) for patients with poor prognostic factors did not demonstrate better outcomes. ${ }^{12,13}$ The exact role and initiation of HDCT and ASCT vary between centers. It is either performed following chemotherapy at first ${ }^{14,15}$ or later recurrences. ${ }^{6,16}$ There is no RCT to substantiate the benefit of HDCT compared with chemotherapy alone in patients with recurrent disease. The ongoing RCT (TIGER trial) is designed to address the benefit of HDCT in patients following the first recurrence after chemotherapy. ${ }^{17,18}$ 
The patients in this series belonged to three different risk groups as per LBS (very low, intermediate, and high risk). All are disease-free till now with good quality of life and regular follow-up. Although the follow-up of the patients was not long enough, no second malignancies were detected.

Different regimens such as high-dose $\mathrm{VIP}^{13}$; high-dose carboplatin, etoposide, and cyclophosphamide (HD-CEC) ${ }^{12}$; high dose carboplatin, etoposide, and thiotepa (HD-CET) ${ }^{19}$; high-dose ifosfamide, carboplatin, and etoposide (HD-ICE) ${ }^{20}$; and high-dose carboplatin, etoposide (HD-CE) have been employed in various studies. Though there are no direct comparisons between these regimens, the outcomes have been similar. The most common HD-CE regimen was used in all the three patients. . $21,22^{2}$

HDCT/ASCT is associated with various hematologic or nonhematologic morbidities. The common toxicities are myelosuppression, mucositis, nausea, vomiting, diarrhea, peripheral neuropathy, and otologic abnormalities. Treatment-related mortality reported in the literature is approximately 3 to $8 \%$ which was primarily due to renal, hepatic, and pulmonary toxicity. ${ }^{6}$ Second malignancies especially acute leukemia had been reported in $<2 \%$ cases. ${ }^{23-25}$ Our study had similar toxicities as described in the literature with no treatment-related mortality or second malignancies.

It is challenging to treat patients with HDCT and ASCT in resource-constrained settings like India. Lack of financial support, lack of centers with the facility to perform HDCT with ASCT, lack of cryopreservation facility for tandem transplants, noncompliance to treatment, and poor stem cell mobilization due to prior multiple lines to treatment are the potential constraints in offering HDCT to GCT patients. Hence, very few centers routinely do HDCT for GCTs, and consequently, there is no published experience from India in this context.

Despite having only three patients with recurrent GCT, our series has shown an excellent survival (100\%) with HDCT and ASCT. The proportion of similar patients who were eligible for HDCT and ASCT during the period was not recorded and contributes to selection bias.

\section{Conclusion}

Though the number of patients reported in this series is limited, HDCT with ASCT seems to be safe and effective in relapsed/refractory testicular GCT with durable complete remission achieved in all three patients. Prospective pooled data of all the relapsed GCT patients from multiple centers would need to be analyzed to further ascertain the exact number of patients who would require HDCT after salvage chemotherapy in India.

\section{Funding}

There were no external sources of funding for this project.

\section{Conflict of Interest}

None of the authors have any relevant conflicts of interest to declare.

\section{References}

1 Albers P, Albrecht W, Algaba F, et al. European Association of Urology. EAU guidelines on testicular cancer: 2011 update. Eur Urol 2011;60(2):304-319

2 van Dijk MR, Steyerberg EW, Habbema JDF. Survival of non-seminomatous germ cell cancer patients according to the IGCC classification: an update based on meta-analysis. Eur J Cancer 2006;42(7):820-826

3 Adra N, Einhorn LH. Salvage therapy for relapsed testicular cancer. Oncotarget 2017;8(41):69200-69201

4 Lorch A, Beyer J, Bascoul-Mollevi C, et al. International Prognostic Factors Study Group. Prognostic factors in patients with metastatic germ cell tumors who experienced treatment failure with cisplatin-based first-line chemotherapy. J Clin Oncol 2010;28(33):4906-4911

5 Lorch A, Bascoul-Mollevi C, Kramar A, et al. Conventional-dose versus high-dose chemotherapy as first salvage treatment in male patients with metastatic germ cell tumors: evidence from a large international database. J Clin Oncol 2011;29(16):2178-2184

6 Einhorn LH, Williams SD, Chamness A, Brames MJ, Perkins SM, Abonour R. High-dose chemotherapy and stem-cell rescue for metastatic germ-cell tumors. N Engl J Med 2007;357(4):340-348

7 Hamid AA, Markt SC, Vicier C, et al. Autologous stem-cell transplantation outcomes for relapsed metastatic germ-cell tumors in the modern era. Clin Genitourin Cancer 2019;17(1):58-64.e1

8 Shamash J, Powles T, Mutsvangwa K, et al. A phase II study using a topoisomerase I-based approach in patients with multiply relapsed germ-cell tumours. Ann Oncol 2007;18(5):925-930

9 Badreldin W, Krell J, Chowdhury S, et al. The efficacy of irinotecan, paclitaxel, and oxaliplatin (IPO) in relapsed germ cell tumours with high-dose chemotherapy as consolidation: a non-cisplatin-based induction approach. BJU Int 2016;117(3):418-423

10 Shanmugalingam T, Soultati A, Chowdhury S, Rudman S, Van Hemelrijck M. Global incidence and outcome of testicular cancer. Clin Epidemiol 2013;5:417-427

11 Saju SV, Radhakrishnan V, Ganesan TS, et al. Factors that impact the outcomes in testicular germ cell tumors in low-middle-income countries. Med Oncol 2019;36(3):28

12 Motzer RJ, Nichols CJ, Margolin KA, et al. Phase III randomized trial of conventional-dose chemotherapy with or without high-dose chemotherapy and autologous hematopoietic stem-cell rescue as first-line treatment for patients with poor-prognosis metastatic germ cell tumors. J Clin Oncol 2007;25(3):247-256

13 Daugaard G, Skoneczna I, Aass N, et al. A randomized phase III study comparing standard dose BEP with sequential high-dose cisplatin, etoposide, and ifosfamide (VIP) plus stem-cell support in males with poor-prognosis germ-cell cancer. An intergroup study of EORTC, GTCSG, and Grupo Germinal (EORTC 30974) Ann Oncol 2011;22(5):1054-1061

14 Motzer RJ, Mazumdar M, Sheinfeld J, et al. Sequential dose-intensive paclitaxel, ifosfamide, carboplatin, and etoposide salvage therapy for germ cell tumor patients. J Clin Oncol 2000;18(6):1173-1180

15 Feldman DR, Sheinfeld J, Bajorin DF, et al. TI-CE high-dose chemotherapy for patients with previously treated germ cell tumors: results and prognostic factor analysis. J Clin Oncol 2010;28(10):1706-1713

16 Vaena DA, Abonour R, Einhorn LH. Long-term survival after high-dose salvage chemotherapy for germ cell malignancies with adverse prognostic variables. J Clin Oncol 2003;21(22):4100-4104

17 Ongoing Clinical Trials in Testicular Cancer. The TIGER Trial. Oncol Res Treat 2016;39(9):553-556 
18 Standard-Dose Combination Chemotherapy or High-Dose Combination Chemotherapy and Stem Cell Transplant in Treating Patients With Relapsed or Refractory Germ Cell Tumors - Full Text View - ClinicalTrials.gov. Available at: https://clinicaltrials.gov/ct2/show/NCT02375204. Accessed April 7, 2020

19 Rick O, Bokemeyer C, Beyer J, et al. Salvage treatment with paclitaxel, ifosfamide, and cisplatin plus high-dose carboplatin, etoposide, and thiotepa followed by autologous stem-cell rescue in patients with relapsed or refractory germ cell cancer. J Clin Oncol 2001;19(1):81-88

20 Selle F, Wittnebel S, Biron P, et al. A phase II trial of high-dose chemotherapy (HDCT) supported by hematopoietic stem-cell transplantation (HSCT) in germ-cell tumors (GCTs) patients failing cisplatin-based chemotherapy: the Multicentric TAXIF II study. Ann Oncol 2014;25(9):1775-1782

21 Simonelli M, Rosti G, Banna GL, Pedrazzoli P; Italian Germ cell cancer Group (IGG). Gruppo Italiano Trapianto Midollo Osseo,
Cellule Staminali Emopoietiche e Terapia Cellulare (GITMO). Intensified chemotherapy with stem-cell rescue in germ-cell tumors. Ann Oncol 2012;23(4):815-822

22 Popovic L, Matovina-Brko G, Popovic M, et al. High dose chemotherapy with stem cell support in the treatment of testicular cancer. World J Stem Cells 2015;7(11):1222-1232

23 OSCarrigan B, Grimison P. Current chemotherapeutic approaches for recurrent or refractory germ cell tumors. Urol Oncol Semin Orig Investig. 2015;33(8):343-354

24 Siegert W, Beyer J, Strohscheer I, et al; The German Testicular Cancer Cooperative Study Group. High-dose treatment with carboplatin, etoposide, and ifosfamide followed by autologous stem-cell transplantation in relapsed or refractory germ cell cancer: a phase I/II study. J Clin Oncol 1994;12(6):1223-1231

25 Nichols CR, Andersen J, Lazarus HM, et al. High-dose carboplatin and etoposide with autologous bone marrow transplantation in refractory germ cell cancer: an Eastern Cooperative Oncology Group protocol. J Clin Oncol 1992;10(4):558-563

\section{Certificate Course in Telemedicine Practice Guidelines}

If you want to get certified in doing Telemedicine Consultation

(as per requirements of Govt of India notification),

please enrol for the six module online course at:

https://www.onference.in/c/certificate-course-in-telemedicine-by-acdh/1610 